\title{
A Case of Pica with Motor-Mental Retardation and with Foreign Bodies in the Larynx and Gastrointestinal Tract: Autopsy Results and Current Literature Review
}

\author{
๑ Omer Faruk Aykir, • Ferhan Kandemir*, • Mehmet Sunay Yavuz, @ Ufuk Akin, ๑ Zehra \\ Zerrin Erkol** \\ Manisa Celal Bayar University Faculty of Medicine, Department of Forensic Medicine, Manisa, Turkey \\ *Ministry of Justice, Council of Forensic Medicine, Kutahya Branch of Forensic Medicine, Kutahya, Turkey \\ ${ }^{* *}$ Abant Izzet Baysal University Faculty of Medicine, Department of Forensic Medicine, Bolu, Turkey
}

\section{Abstract}

Pica is the continuous consumption of non-nutritive and non-food items for at least one month. In this study, a 17-year-old boy pica case diagnosed with motor-mental retardation due to cerebral palsy was presented. It was learned that the case was exhibiting object swallowing behaviour. He was found dead in the field by his relatives. At the autopsy, a $3.5 \times 3.0 \times 2.5 \mathrm{~cm}$ foreign body (stone) that completely obstructed the upper part of the larynx lumen was removed. In addition, three foreign bodies were removed from the cecum region. The death was caused by mechanical asphyxia due to foreign body aspiration.

Keywords: Pica, foreign bodies, asphyxia, intellectual disability, autopsy

\section{Introduction}

According to "DSM- 5 Diagnostic Criteria for Eating Disorders", pica is the continuous consumption of nonnutritive and non-food items for at least one month (1). The consumption of non-food products is harmful to the health of the individual and the development of children. In addition, eating non-nutritious substances is not culturally or socially appropriate. Eating behavior may be severe enough to require additional clinical intervention if it occurs due to mental disorders such as mental retardation, autism spectrum disorder, schizophrenia, or another medical condition such as pregnancy (1). The most common forms of pica are geophagia (clay, sand, mud, soil), pagophagia (ice) and trichophagia (hair) (2). Lithobezoar is the accumulation of stones and is commonly associated with a history of pica (3). Pica may be common in young children with autism spectrum disorder and intellectual disability (4). Persons with developmental and intellectual disabilities are at risk of foreign body aspiration, because a large percentage of this population is affected by dysphagia, pica (5).
In this study, a pica case diagnosed with mental retardation was presented, the importance of detailed autopsy in death cases due to foreign body aspiration was emphasized and it was aimed to draw attention to foreign body aspiration in individuals with mental retardation.

\section{Case Report}

Ethics approval for the study was obtained from Bolu Abant Izzet Baysal University Clinical Researches Ethics Committee Approval dated May 29, 2020 and numbered 168. Permission was obtained from the father of the case for the case report.

A 17-year-old boy was found dead in the field by his relatives. It was learned that the case was receiving treatment for motor-mental retardation due to cerebral palsy, and exhibited object swallowing behavior while not using medication.

External examination of the case revealed that there was soil transmission around his mouth and nose. No traumatic lesions were detected. The pre-autopsy radiological

Address for Correspondence: Zehra Zerrin Erkol, Abant Izzet Baysal University Faculty of

Medicine, Department of Forensic Medicine, Bolu, Turkey

E-mail: zerrinerko|@gmail.com ORCID: orcid.org/0000-0003-0281-9389

Received: 18.05.2021 Accepted: 30.05.2021

Copyright 2021 by The Medical Bulletin of istanbul Haseki Training and Research Hospital The Medical Bulletin of Haseki published by Galenos Yayınevi. 
examination revealed a radiopaque appearance on the upper part of the trachea and a metallic radiopaque appearance on the right lower quadrant of the abdomen (Figure $1 \mathrm{a}$ and $1 \mathrm{~b}$ ).

The autopsy revealed hyperemia in the epiglottis, ecchymotic areas adjacent to the epiglottis and the junction of both vocal cords. During dissection, a $3.5 \times 3.0 \times 2.5 \mathrm{~cm}$ foreign body (stone) that completely obstructed the upper part of the larynx lumen was removed (Figure 2a). In addition, one $9 \mathrm{~cm}$ long ice-cream stick, one $10 \mathrm{~cm}$ long metal object (crochet) and one $10.5 \mathrm{~cm}$ long branch were removed from the cecum region (Figure $2 \mathrm{~b}$ and $2 \mathrm{c}$ ). There were no macroscopic pathological features observed in the other organs. It was detected that the death was caused by mechanical asphyxia due to foreign body aspiration.
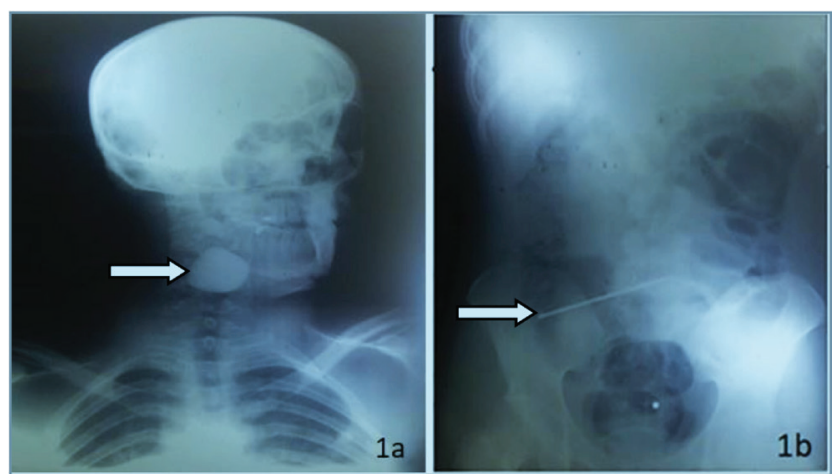

Figure 1. Radiopaque view of foreign body (stone) in neck region (1a) and metal object (crochet) in abdominal region (1b)
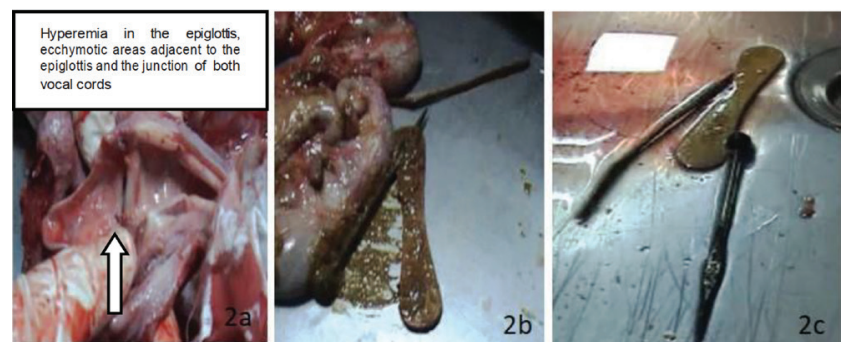

Figure 2. The appearance of the upper part of the larynx where the foreign body was removed (2a) and three foreign bodies removed from the cecum area (ice cream stick, branch piece and crochet) (2b-2c)

\section{Discussion}

A foreign body in the respiratory tract is an important and serious event. Injuries caused by foreign substances in the respiratory tract seriously threaten the health and life of children (6). In our case, who was a 17-year-old child, it was stated by his relatives that he had motor-mental developmental retardation due to cerebral palsy, and he had a habit of eating foreign bodies such as soil, stone and wood. At the autopsy, it was determined that case's death was occurred due to mechanical asphyxia caused by a stone block that completely occluded the lumen within the larynx.

One of the most serious complications that may develop in cases with pica history is an intestinal obstruction (7). Although three foreign bodies were found in the cecum region of our case, there was no history of obstruction. The risk of developing intestinal obstruction should be considered in cases with pica. Numanoğlu and Tatli (8) have reported that there was iron deficiency anemia in a 4-yearold male pica case and partial intestinal obstruction was developed. The case has been treated with the removal of foreign bodies, mostly stones via anal dilatation, enema and touch, under general anesthesia, and no laparotomy was required (8).

The presented case shows the importance of obtaining detailed information about the medical history, habits, drugs used and the development of the event from the relatives of the deceased before the autopsy. Crime scene investigation, radiological examinations of the entire body of the deceased before the autopsy and learning from the family of the deceased that the victim had a habit of eating foreign objects that were not food, provided important data for forensic medicine specialists before the autopsy. The case also emphasizes the importance of careful and detailed examination of the respiratory tract and gastrointestinal tract in order to determine whether there are other swallowed foreign bodies in corpses where a foreign body causing obstruction in the respiratory tract is detected.

In the literature, it has been reported that the morbidity rate and mortality rate are $34-40 \%$ and 11 $40 \%$ respectively in pica cases with mental retardation who underwent laparotomy with gastrointestinal tract problems (9). In addition to the high morbidity and mortality rates, when respiratory aspirations are added, as in our case, serious mortality rates are encountered and treatment becomes more important in these cases. In a study, it has been stated that $80 \%$ success was achieved in the treatment of pica with cognitive-behavioral treatment together with intensifier and response reduction method in mentally retarded individuals (10).

As a result, since they cannot fully express their complaints, mental retardation cases are the patient groups that needed to be evaluated clinically in detail. Considering that the pica is more common in patients with mental retardation diagnosis, we think that laboratory and radiological examinations should be evaluated in detail in 
these cases, necessary treatments should be provided, and thus possible mechanical asphyxia and intestinal obstruction and related deaths may be prevented.

\section{Authorship Contributions}

Concept: O.F.A., F.K., Design: O.F.A., F.K., Data Collection or Processing: O.F.A., F.K., M.S.Y., U.A., Z.Z.E., Analysis or Interpretation: F.K., M.S.Y., U.A., Z.Z.E., Literature Search: F.K., M.S.Y., U.A., Writing: O.F.A., F.K., M.S.Y., U.A., Z.Z.E.

Conflict of Interest: No conflict of interest was declared by the authors.

Financial Disclosure: The authors declared that this study received no financial support.

\section{References}

1. Institute for Eating Disorders. DSM- 5 Diagnostic Criteria for Eating Disorders: https://insideoutinstitute.org.au/assets/ dsm-5\%20criteria.pdf (Avaible on: 28.05.2021).

2. Canturk N, Ozdes T, Dogan B. Pica: a case report. J For Med 2010;24:62-7.

3. Aihole JS. Giant Colonic Lithobezoar: A Rare Case Report. J Indian Assoc Pediatr Surg 2020;25:103-5.
4. Fields VL, Soke GN, Reynolds A, et al. Pica, Autism, and Other Disabilities. Pediatrics 2021;147:e20200462.

5. Yocum AD, Dennison JL, Simon EL. Esophageal Obstruction and Death in a Nonverbal Patient. J Emerg Med 2021;60:e109-e13.

6. Liu J, Wang Y, Zhao T, Li C, Nie L. Application of 64-Slice Spiral CT Imaging Technology Based on Smart Medical Augmented Reality in the Diagnosis of Foreign Bodies in the Respiratory Tract in Children. J Healthc Eng 2021;2021:9962997.

7. Tripodi V, Caruso A, Luraghi $M$, et al. Paediatric gastric trichobezoar: the Rapunzel syndrome A report of two cases. Ann Ital Chir 2020;9:S2239253X20032442.

8. Numanoğlu KV, Tatli D. A rare cause of partial intestinal obstruction in a child: colonic lithobezoar. Emerg Med J 2008;25:312-3.

9. Miyakawa K, Ito M, Hatta K, Eto K, Arai H. Recurrent cholecystitis in an elderly mentally retarded patient with pica. Psychogeriatrics 2011;11:244-6.

10. Hagopian LP, Rooker GW, Rolider NU. Identifying empirically supported treatments for pica in individuals with intellectual disabilities. Res Dev Disabil 2011;32:2114-20. 